\title{
Fault Diagnosis of System-Level Equipment with a Deep Learning Framework
}

\author{
Kexuan Wang ${ }^{1}$, Chunyang Zeng ${ }^{2}$ and Xiaoliang $\mathrm{Zhu}^{1, \mathrm{a}}$ \\ ${ }^{1}$ School of Energy and Environment, Southeast University, Nanjing, China \\ ${ }^{2}$ School of Energy and Environment, Southeast University, Nanjing, China
}

\begin{abstract}
In this paper, a deep learning-based fault diagnosis framework is proposed to improve the fault diagnosis accuracy of system-level equipment such as condenser systems in nuclear power plants. The condenser system signals are non-vibrating, slowly time-varying, and multi-dimensional in nature. Therefore, in this paper, we propose a deep learning-based fault diagnosis framework, which adopts the idea of combining data warehouse modeling and deep learning fault diagnosis, and establishes the data set required for deep learning through accurate simulation modeling of typical condenser faults, so as to make full use of the feature extraction capability of deep learning under large-scale samples. Based on this, an end-to-end deep learning model is developed for accurate diagnosis of multiple condenser faults under multiple system conditions. Through the fault diagnosis experiments on the validation set data under various system conditions, the fault diagnosis accuracy is as high as 0.9584 , which verifies the effectiveness of the proposed framework in fault diagnosis of system-level equipment.
\end{abstract}

\section{Introduction}

The condenser system is a major subsystem of the nuclear two-loop system, and the safe and efficient operation of the condenser system is of great importance to the safety and economy of nuclear power units. However, there are many typical faults in the condenser system that are difficult to detect or predict in a timely manner, which can lead to a decrease in the economics of the nuclear unit and even jeopardize the safe operation of the unit. Accurate fault diagnosis of the condenser system can significantly improve the continuous and reliable operation of nuclear power plants, avoid major faults as much as possible, and reduce the efficiency losses caused by faults.

There are three main types of existing fault diagnosis techniques for system-level equipment, namely modelbased techniques, data-based techniques, and experiencebased techniques. Model-based techniques identify, locate and isolate faults by abstracting functional relationships consisting of coupled mathematical equations that can describe system functions [1]. However, accurate mathematical models of large systems are difficult to establish, making it difficult to use model-based techniques. Empirical-based fault diagnosis techniques use the knowledge in existing real-time databases and the rules in rule bases to diagnose faults. However, empiricalbased fault diagnosis techniques are limited by the human ability to understand the system and can only diagnose the more obvious faults.

On the other hand, data-based techniques, represented by deep learning, can enable fault diagnosis without being limited by human understanding of the system. Deep learning can extract multiple statistical parameters to represent the health state of the system by statistically analyzing the actual unit operation data, and then train classifiers to diagnose faults.

In recent years, deep learning methods have been widely applied to fault diagnosis in the nuclear power industry. For example, Shi [2] used a fuzzy neural network-based approach for fault identification for typical faults arising during steam generator operation, and achieved good results. Zhou Gang et al [3] proposed an integrated neural network method applied to the fault diagnosis of steam generators, which combined predictors and classifiers to improve the monitoring and diagnosis of steam generator faults. Lin Xiaogong et al [4] proposed a genetic neural network for fault diagnosis of steam generators in response to the shortcomings of traditional BP neural networks that are easily trapped in local minima and slow convergence speed.

However, the scarcity of fault data in industrial sites has led to traditional deep learning models limited by the amount of data and low accuracy. Therefore, it is necessary to develop a new deep learning method for solving the scarcity of field data and thus improving the fault diagnosis accuracy for system-level equipment.

In this paper, a deep neural network-based fault diagnosis framework is proposed to achieve effective diagnosis for a wide range of condenser faults. The main contributions are summarized as follows.

- Apros-based condenser simulation model for realizing a variety of condenser faults

- A deep neural network model that enables

a Xiaoliang Zhu: zx113794521@163.com 
effective feature extraction for non-oscillating, slowly time-varying, multi-dimensional signals for effective identification of condenser faults.

The rest of this paper is organized as follows. Section II briefly introduces the fault diagnosis model framework, details the Apros condenser simulation modality modeling method, and briefly describes the basic deep neural network model structure and composition. Section III gives the modeling results and fault diagnosis results. Section IV gives the conclusion.

\section{Methods and Meterials}

\subsection{Fault diagnosis model framework}

This paper investigates the fault diagnosis of condenser systems in nuclear power units. Taking the N-3560-2 condenser of Huaneng Suzhou Thermal Power Plant as the target, this paper investigates the following five typical condenser system faults [5], including:

1) Decrease in efficiency of circulating water pump

2) Decreased sealing performance

3) Heat transfer deterioration

4) Reduced steam pumping performance

5) Leakage into high temperature vapor source

The existing arrangement of condenser sensors in nuclear power units is not yet able to directly monitor the above five faults, so in this paper, we consider an actual nuclear power unit condenser system measurement point arrangement, while referring to the actual arrangement specification of nuclear power units, and select 31 measurement variables with actual monitoring capability to reflect the condenser system state when the above five faults occur. Based on this, a fault diagnosis framework based on deep learning is proposed in this paper, as shown in Figure 1.

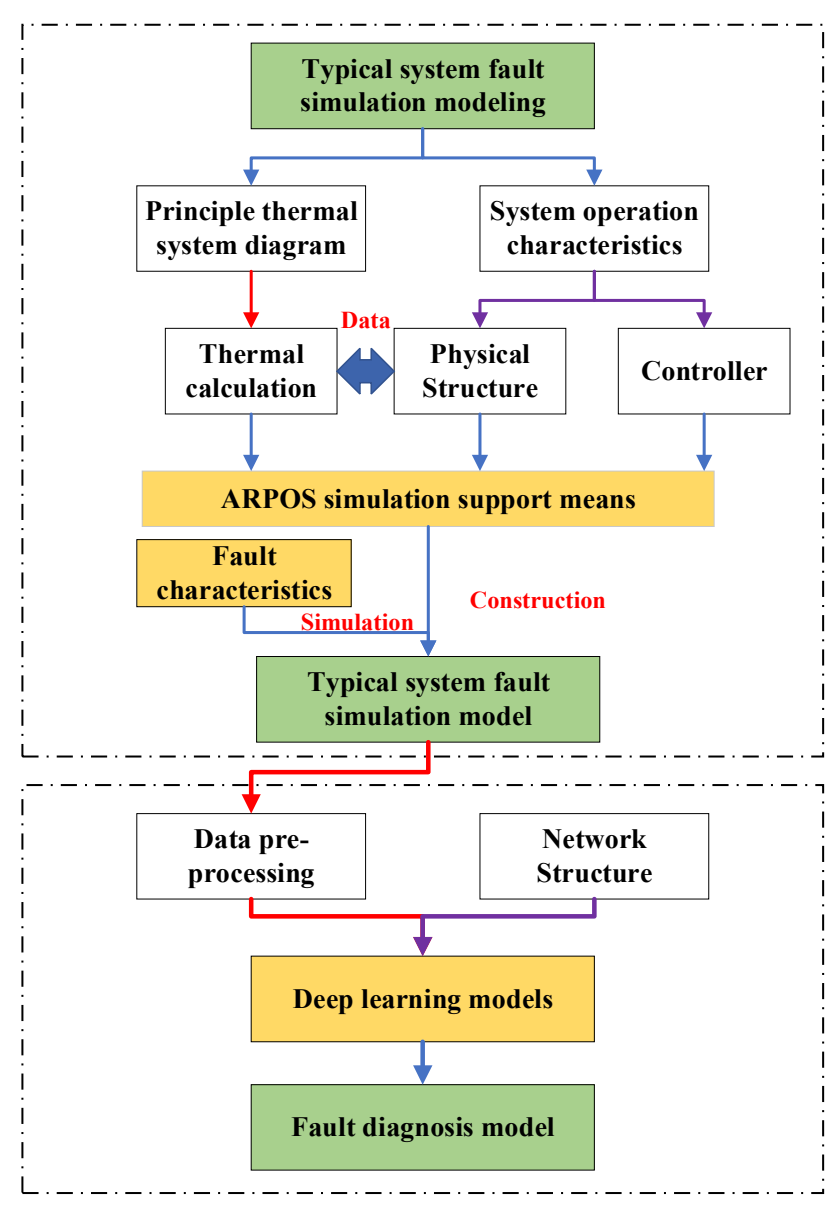

Fig 1. Deep learning - based fault diagnosis framework

\subsection{Apros-based fault model for condenser systems}

Apros is a powerful, forward-thinking advanced process design and simulation software developed by Fortum and VTT Technical Research Centre of Finland. It is used for the design and optimization of process systems and automation systems, the development of operating systems and training systems, and the full range of dynamic and accident analysis.
The Apros simulation platform is used to build a condenser system model for nuclear power plants and to realize steady-state and dynamic simulations of condenser systems. Five fault characteristics of the condenser are considered separately, and measurable parameters that can correctly reflect the fault characteristics are found to simulate condenser faults on the established Apros simulation model.

Then, we simulate the above five fault conditions, collect 31 measurement variables with actual 
measurement significance as input, and obtain the corresponding state of the condenser system by automatically setting the fault occurrence time. Thus, an end-to-end deep neural network model is built for the diagnosis of condenser system fault conditions.

\subsection{Neural network models for fault diagnosis}

A deep neural network is a deep learning framework consisting of a multilayer perceptron that extracts highlevel features from raw data using statistical learning methods to obtain an effective representation of the input space in a large amount of data. As shown in the figure, a deep neural network has four components: nonlinear neurons, forward propagation, backward propagation, and softmax. The following concepts will explain these basic concepts.

A neuron is a nonlinear input-output model that constructs a nonlinear relationship between input and output, and it aims to fit the corresponding mapping relationship between input and output. The basic neuron model can be represented as:

$$
\begin{gathered}
z=\sum_{i=1}^{m} w_{i} x_{i}+b \\
\text { output }=\operatorname{sign}(z)
\end{gathered}
$$

where $z$ denotes the output value of linear transformation, $\mathrm{w}$ denotes the coefficient of linear relationship, $x$ denotes the input value, $b$ denotes the offset value, output denotes the output value, and $\operatorname{sign}()$ denotes the activation function, which is used for nonlinear transformation. The common activation functions include sigmoid, tanh, and ReLU. the ReLU function is the most commonly used activation function for deep learning models, which can effectively prevent the gradient of deep neural networks from vanishing. Since the derivative of the ReLU function is either 1 or 0 , the range of the features is not easily changed when they are passed between layers. the ReLU function is represented by the following equation.

$$
\operatorname{ReLU}(x)= \begin{cases}x & \text { if } x>0 \\ 0 & \text { if } x \leq 0\end{cases}
$$

The forward propagation mechanism is a series of linear and activation operations using several weight coefficient matrices $\mathrm{W}$, bias vectors $\mathrm{b}$ and input values $\mathrm{x}$. Starting from the input layer, one layer is computed backwards until the output layer, and the output value is obtained. The specific algorithm is represented as follows.

Algorithm1: Forward Propagation

$$
\begin{aligned}
& \text { 1. initialize } a^{1}=x \\
& \text { 2. for } l=2 \text { to } L \\
& a^{l}=\sigma\left(z^{l}\right)=\sigma\left(W^{l} a^{l-1}+b^{l}\right) \\
& \text { 3. output }=a^{L}
\end{aligned}
$$

Where, $a$ denotes the output value, $l$ denotes the number of neural network layers, $L$ denotes the total number of neural network layers, $\sigma$ and denotes the nonlinear activation function.

The back propagation mechanism is to find the error value obtained by comparing the output value with the sample, and then use the gradient descent method to bias the error function and finally update the parameters of the whole neural network, i.e., the weight coefficient matrix $\mathrm{W}$ and the bias vector $\mathrm{b}$. The specific algorithm is expressed as follows.

Algorithm2: Back propagation

1. Calculate the total error

$$
J(W, b, x, y)=\frac{1}{2}\left\|a^{L}-y\right\|_{2}^{2}
$$

2. Calculate the output layer

$$
\begin{aligned}
& \frac{\partial J(W, b, x, y)}{\partial W^{L}}=\left(a^{L}-y\right)\left(a^{L-1}\right)^{T} \odot \sigma^{\prime}(z) \\
& \frac{\partial J(W, b, x, y)}{\partial b^{L}}=\left(a^{L}-y\right) \odot \sigma^{\prime}(z)
\end{aligned}
$$

3. Define $\delta^{l}=\frac{\partial J(W, b, x, y)}{\partial z^{l}}$

4. Recurrence relation

$$
\delta^{l}=\delta^{l+1} \frac{\partial z^{l+1}}{\partial z}=\left(W^{l+1}\right)^{T} \delta^{l+1} \odot \sigma^{\prime}\left(z^{l}\right)
$$

5. Update weights

$$
W^{l}=W^{l}-\alpha \sum_{i=1}^{m} \delta^{i, l}\left(a^{i, l-1}\right)^{T}
$$

6. $\quad b^{l}=b^{l}-\alpha \sum_{i=1}^{m} \delta^{i, l}$

7. Output $W$ and $b$

where, $J(W, b, x, y)$ denotes the loss function, which is used to measure the goodness of the training result; $y$ denotes the state of the condenser system; $\alpha$ denotes the learning rate.

Softmax is used in the multi-classification process, which maps the output of multiple neurons into the $(0,1)$ interval to perform multi-classification. the Softmax function is defined as

$$
\operatorname{Softmax}\left(z_{i}\right)=\frac{e^{z_{i}}}{\sum_{c=1}^{C} e^{z_{c}}}
$$

The classification accuracy of the Softmax classifier can be calculated by the following equation:

$$
\text { accuracy rate }=\frac{\text { correctly classified data size }}{\text { test data size }}
$$

\section{Results}

\subsection{Apros-based fault model for condenser systems}

Two parts are required to build an accurate Apros condenser simulation model, one is to perform structural thermal calculations, and the other is to model the called turn, i.e., thermal calculations compared with simulation, and through operational experiments compared with simulation. Based on the main structural parameters of the condenser shown in Table 1, the Apros condenser 
simulation model is built.

Table1. Condenser parameter table

\begin{tabular}{|c|c|c|}
\hline Model & & $\mathrm{N}-3560-2$ \\
\hline Item & Unit & Parameter \\
\hline Condenser pressure & $\mathrm{kPa}$ & 4.34 \\
\hline $\begin{array}{l}\text { Cooling water } \\
\text { temperature }\end{array}$ & ${ }^{\circ} \mathrm{C}$ & 20 \\
\hline Cooling water & $\mathrm{m}^{3} / \mathrm{h}$ & 10000 \\
\hline $\begin{array}{l}\text { Cooling water } \\
\text { temperature rise }\end{array}$ & ${ }^{\circ} \mathrm{C}$ & 6.5 \\
\hline Cooling water flow & - & 2 \\
\hline $\begin{array}{c}\text { Cooling water } \\
\text { velocity in the tube }\end{array}$ & $\mathrm{m} / \mathrm{s}$ & 2.06 \\
\hline $\begin{array}{c}\text { Condenser heat } \\
\text { load }\end{array}$ & $\mathrm{kJ} / \mathrm{s}$ & 75325 \\
\hline Cleaning factor & - & 0.82 \\
\hline $\begin{array}{l}\text { Condenser water } \\
\text { resistance }\end{array}$ & $\mathrm{kPa}$ & 38.40 \\
\hline $\begin{array}{l}\text { Condensate tube } \\
\text { specifications }\end{array}$ & $\begin{array}{l}\text { Condensation } \\
\text { zone } \\
\text { Air-cooled } \\
\text { zone }\end{array}$ & $\begin{array}{c}\varphi 25.4 \times 1 \mathrm{~mm} \\
\mathrm{HSn} 70-1 \mathrm{~B} 5610 \\
\varphi 25.4 \times 0.6 \mathrm{~mm} \\
\text { TA2 } 634\end{array}$ \\
\hline $\begin{array}{l}\text { Effective length of } \\
\text { condenser tube }\end{array}$ & $\mathrm{mm}$ & 7150 \\
\hline $\begin{array}{c}\text { Tube plate } \\
\text { material/thickness }\end{array}$ & $\mathrm{mm}$ & Q235-A-0/30 \\
\hline
\end{tabular}

The condenser simulation model built in this paper achieves the basic fitting of the temperature-flow curves of the condenser under three design conditions of $100 \mathrm{t} / \mathrm{h}$, $150 \mathrm{t} / \mathrm{h}$ and $200 \mathrm{t} / \mathrm{h}$, as shown in Figure 2.

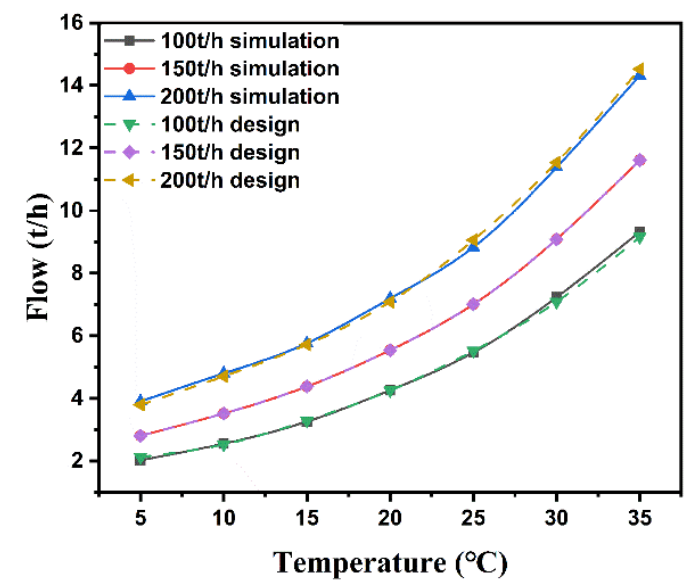

Fig 2. Condenser Simulation - Design Curve

Taking the condenser vacuum leakage fault as an example, the simulation operation curve basically fits the actual vacuum leakage data, as shown in Figure 3.

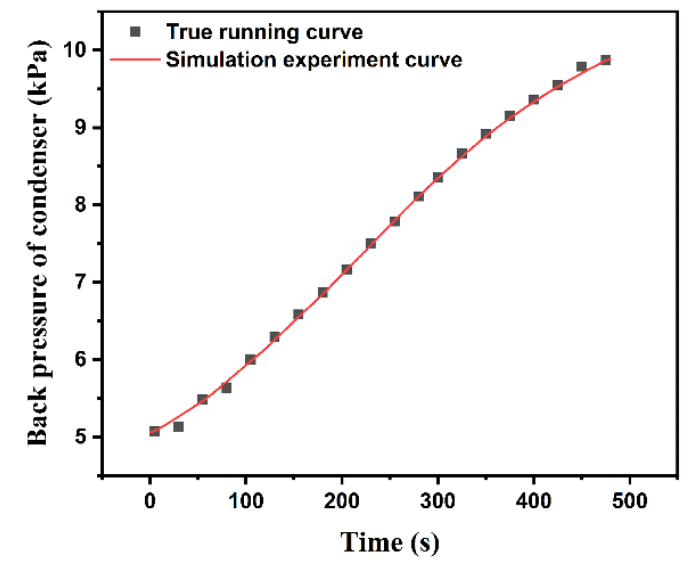

Fig 3. Condenser simulation - actual fault characteristic curve

\subsection{Deep Neural Network Model}

The DNN model developed in this paper was implemented using Keras, an open source artificial neural network library written in Python. The experiments were conducted on a computer with an AMD R7-4800H CPU and an Nvidia MX450 GPU. The results of the experiments are discussed in this section.

A 6-layer DNN is used to implement the fault diagnosis function. The DNN contains a 31-dimensional input layer, 4 hidden layers, and a 6-dimensional output layer, and the final output value is output to a softmax function to solve the probability value to obtain the state of the condenser system.

By collecting 6 sets of data containing 5 fault states and normal states in the APROS condenser simulation model, each set contains 600 s of system state data, and finally selecting $90 \%$ of them as the training set and $10 \%$ as the test set, the final experimental results were obtained as shown in Figure 4 and Figure 5.

Five typical load points, seven typical ambient temperature points, and four different fault severity levels corresponding to five fault states are taken as repeated experimental data, and the final validation set has a total of 700 sets of fault experimental data. The accuracy of the trained deep neural network fault model is verified, and the accuracy of the SVM method for the above fault data is also compared, and the results are shown in the following table.

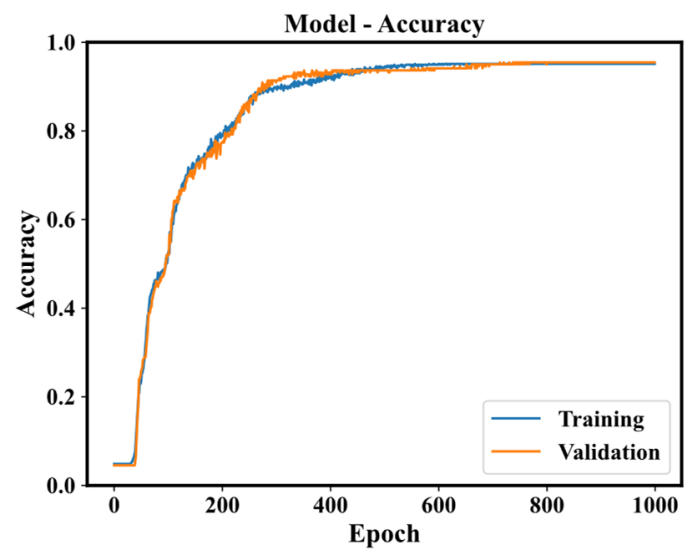

Fig 4. Neural network accuracy curve 


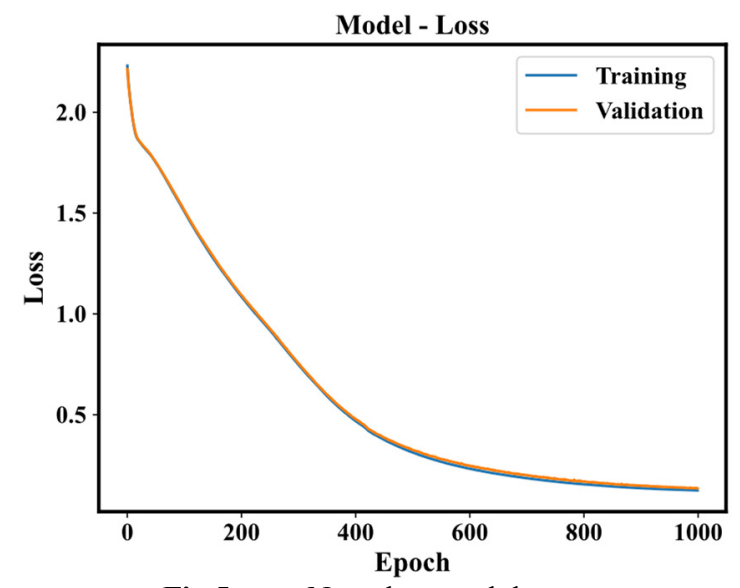

Fig 5. Neural network loss curve

Table2. Model Accuracy

\begin{tabular}{cc}
\hline Model & Accuracy \\
\hline DNN & 0.9584 \\
SVM & 0.9217 \\
\hline
\end{tabular}

As shown in Table 3, the accuracy of the deep neural network model for fault diagnosis is as high as 0.9584 , while the accuracy of the traditional fault diagnosis method SVM is only 0.9217 . Obviously, the deep neural network model has a higher accuracy for fault diagnosis of system-level equipment.

\section{Conclusion}

It is of great importance to apply deep learning to fault diagnosis of system-level equipment with non-vibrating, slowly time-varying, multi-dimensional signals. In this paper, a deep learning-based fault diagnosis framework for nuclear power condenser systems is proposed. That is, a simulation model of a real condenser system is built based on the Apros simulation platform, and an accurate simulation of a typical condenser fault is performed on this basis to build a data warehouse required for deep learning. A deep neural network model is built to realize the identification of multiple fault states of complex systemlevel equipment in the context of multidimensional data.

The developed fault diagnosis system, in which the temperature-flow characteristic curves of the condenser system simulation model under three design conditions including $100 \mathrm{t} / \mathrm{h}, 150 \mathrm{t} / \mathrm{h}$ and $200 \mathrm{t} / \mathrm{h}$ are basically fitted with the actual data; taking the condenser vacuum leakage fault as an example, the simulation operation curves are basically fitted with the actual vacuum leakage data. Therefore, the condenser simulation model can establish a reliable data warehouse for deep learning.

In terms of fault diagnosis accuracy, the developed deep learning-based fault diagnosis model can achieve a fault diagnosis accuracy of up to 0.9584 . The traditional SVM method, on the other hand, has a maximum fault diagnosis accuracy of 0.9217 due to its difficulty in handling the high dimensional data of system-level equipment. Therefore, the fault diagnosis model based on deep learning greatly improves the fault diagnosis accuracy of system-level equipment.
The developed fault diagnosis system can be widely used in the fault diagnosis process of industrial systemlevel equipment, and its data warehouse-deep neural network framework can realize the digital operation of industrial systems.

\section{References}

1. Y Zhao, Q Zhang and C L Dong. Design and implementation of nuclear power plant fault diagnosis expert system based on DUCG[J]. Atomic Energy Science and Technology, 2016.

2. J Shi, et al. Default diagnosing system of steam generator for nuclear power plant[J]. Journal of Harbin Engineering University, 2001.

3. G Zhou, Y Li. Application of Integrated Neural Network Method to Fault Diagnosis of Nuclear Steam Generator[J]. Atomic Energy ence and Technology, 2009.

4. X G Lin, X W Jiang, T Liu, et al. Application of genetic neural network in steam generator fault diagnosing[J]. Nuclear Power Engineering, 2005.

5. L D Gu. Application and research of energy-saving technology in urban thermal power plants[D]. Southeast University, 2018. 